\title{
sciendo
}

\section{The Influence of Verbal Instruction on Measurement Reliability and Explosive Neuromuscular Performance of the Knee Extensors}

\author{
by \\ Hamdi Jaafar ${ }^{1,2}$, Hanene Lajili ${ }^{3}$
}

\begin{abstract}
The current study aimed to examine the effect of verbal instruction on explosive force production and betweensession measurement reliability during maximal voluntary contractions of knee extensors. Following familiarization, 20 healthy males performed 3 maximal contractions with a "hard-and-fast" instruction and 3 maximal contractions with a "fast" instruction during 2 test-retest sessions. Knee extension maximal voluntary force $\left(F_{\max }\right)$ and the maximal rate of force development $\left(R F D_{\max }\right)$ were measured. Maximal electromechanical delay $\left(E M D_{\max }\right)$, and the maximal rate of muscle activation (RMA max) of quadriceps muscles were determined. No significant effect of instruction was observed on $F_{\max }(p>0.05)$. The RFD $D_{\max }$ and RMA max were significantly higher with the "fast" compared to the "hard-and-fast" instruction $\left(36.07 \%, E S=1.99\right.$ and $37.24 \%, E S=0.92$, respectively), whereas $E M D_{\max }$ was significantly lower with the "fast" instruction compared to the "hard-and-fast" instruction $(-3.79 \%, E S=-0.29)$. No significant differences between test and retest measurements were found $(p<0.05)$. However, the reliability of the RFD max was higher with the fast instruction compared to the hard-and-fast instruction (CV: 7.3 vs. $16.2 \%$; ICC: 0.84 vs. 0.56). Besides, the RFD max was associated with the RMA max and EMD max with a significant effect of instruction. Data showed that the instruction given prior contracting muscle affected explosive force production and associated neuromuscular variables. As a result, the "fast" instruction may be preferred in the assessment of explosive force capacity of skeletal muscle during maximal efforts.
\end{abstract}

Key words: maximal force, force development, electromyography, neuromuscular performance.

\section{Introduction}

The explosive contraction of skeletal muscles is fundamental in many sports activities such as sprinting, jumping, throwing and cycling. Skeletal muscle function can be assessed with the use of dynamic or isometric voluntary contraction protocols. Of particular importance when assessing the maximal voluntary contraction (MVC) two attributes are generally determined: the subject's maximal force generating capacity, i.e., maximal voluntary force $\left(F_{\max }\right)$, and the maximal rate at which force can be developed, i.e., the maximal rate of force development (RFD $\left.{ }_{\max }\right)$ (Maffiuletti et al., 2016).
Some authors have reported a strong positive relationship between the RFD $\max$ and $\mathrm{F}_{\max }$ achieved during MVCs (Bellumori et al., 2011; Van Cutsem et al., 1998; Gołaś et al., 2016). Other research studies, however, have shown that the verbal instruction given by the investigator influenced RFDmax measurement (Bozic et al., 2012; Christ et al., 1993; Holtermann et al., 2007; Sahaly et al., 2001; Stastny et al., 2015). For instance, the recorded RFDmax differed when subjects were asked to exert muscle force in a "fast" or in a "hard-and-fast" fashion. Indeed, the RFD max was significantly higher when subjects were instructed to exert muscle force in a "rapid"

1 - Institut du Savoir Montfort - Recherche, Ottawa, Canada.

2 - Faculty of Medicine, Department of Biochemistry, Microbiology and Immunology, University of Ottawa, Ottawa, Canada.

3 - Centre de Rééducation et de Réadaptation Fonctionnelle La Châtaigneraie, Menucourt, France. 
fashion with no concern for $F_{\max }$ than when they were asked to exert muscle force in a "strong and rapid" fashion (Sahaly et al., 2001, 2003).

Explosive force production is a byproduct of a neural drive from the central nervous system and intrinsic muscle properties (Johnson et al., 2015). Consequently, the rate of muscle activation (RMA), which defines the slope of the EMG activity-time curve in the earlier phase of force development (Inglis et al., 2013; Mitchell et al., 2011), and the electromechanical delay (EMD), the time between muscle activation and muscle force generation (Zhou et al., 1995, 1996), are important descriptors of neuromuscular performance during MVCs. These two attributes may be affected by the instruction when assessing subject's explosive force generating capacity.

The literature revealed conflicting findings regarding the relevance of the relationship between isometric testing and dynamic performance capacity (Juneja et al., 2012). One possible rationale for the conflicting relationship between isometric force assessment and dynamic activities or dynamic force measurement results could be related to the effect of instruction on the reliability of the measurements. The authors know of no study that has examined the impact of the instruction on the reliability of explosive force production and associated neuromuscular variables. Such observations may enhance our understanding regarding neuromuscular strategies during MVCs and provide important implications for neuromuscular testing and training regimens with practical considerations for both athletes and practitioners. Thus, we designed the current study aiming to examine the effect of the instruction on the reliability of neuromuscular variables during MVCs of knee extensors. Additionally, the impact of the instruction on the relationships between the $\mathrm{RFD}_{\max }$ and neuronal variables was evaluated to determine which instructions induced the greatest outcome.

\section{Methods}

\section{Participants}

Twenty healthy males were recruited to participate in this study. The participants' mean $( \pm$ standard deviation) age, body height and body mass were $23.45 \pm 1.99$ years, $1.83 \pm 0.05 \mathrm{~m}$ and $77.79 \pm 4.85 \mathrm{~kg}$, respectively. All participants were physically active and regularly engaged in aerobic and resistance exercises as part of their training regimens. The participants had no (i) current injury to the back, upper or lower limbs; (ii) orthopedic lower limb injury in the past six months; or (iii) history of neurological disorders or lower limb ligament surgery. Each participant completed an informed written consent form before in the commencement of the study. The study was approved by the Institutional Review Board of the Rehabilitation Centre and conducted in accordance with the Declaration of Helsinki.

\section{Experimental procedures}

We designed a cross sectional study in order to assess the effects of 2 different verbal instructions on the reliability of the various variables related to explosive force production during the MVC of knee extensors. A week prior to the experimental period, all of the participants visited the laboratory to familiarize themselves with the testing apparatus and specific requirements, performing several MVCs with different instructions. Then, the participant performed two experimental sessions at the same time of day to avoid the effects of diurnal variations (Jaafar and Lajili, 2018), two-seven days apart. All testing was performed in a room at constant temperature and relative humidity (mean \pm SD: $22 \pm 0.1^{\circ} \mathrm{C}$ and $40 \pm 0.4 \%$, respectively) in order to minimize potential effects of ambient temperature changes on neuromuscular performance (Bell, 1993). The participants were instructed to adhere to an identical pretest protocol: to refrain from intense physical activity for 48 hours before the experimental session and to maintain their usual sleeping habits on the night preceding each experimental session. The participants performed a specific light warm-up consisting of five minutes of cycling on a cycle ergometer (Monark $828 \mathrm{E}$, Vansbro, Sweden).

\section{Electrode Placement}

Recording electrodes were placed according to the European recommendations for surface EMG (Hermens et al., 2000). The skin under the electrode was shaved to remove hair and dead cells, treated with abrasive gel and cleaned with isopropyl alcohol to achieve interelectrode impedance below $2 \mathrm{k} \Omega$. The EMG signals were collected by a pair of adhesive bipolar surface electrodes (FIAB, Florence, Italy) 
attached to the dominant leg. The electrodes were fixed lengthwise on the skin and parallel to the direction of the muscle fibers at an inter-electrode distance of $20 \mathrm{~mm}$. The electrodes were placed at approximately $2 / 3$ of the distance between the anterior superior iliac spin and the lateral aspect of the patella for the vastus lateralis, at approximately $4 / 5$ of the distance between the medial gap of the knee joint and the anterior superior iliac spine for the vastus medialis, and at $1 / 2$ of the distance between the anterior superior iliac spine and the superior border of the patella for the rectus femoris (Hermens et al., 2000). The electrode placement sites were determined visually during a resisted isometric contraction, and were marked with an indelible felt-tip pen to allow repeated measurements from the same muscle area in the subsequent session. The reference electrode was placed over the lateral right malleolus.

\section{Apparatus and testing position}

The participant was seated on a padded chair designed for isometric knee extension force testing (Kin-Com, Chattanooga, TN, USA). The participant's thighs, pelvis, and trunk were pressed tightly against the chair using $5-\mathrm{cm}$ wide non-elastic straps to minimize any extraneous movement of the muscle group of interest. A calibrated strain-gauge force transducer (Interface Inc., Scottsdale, AZ, USA) was coupled to the participant's dominant leg by a non-elastic band positioned just above the lateral malleolus. The knee and hip joints were fixed at $90^{\circ}$. The rigid interface between the transducer and the leg provided virtual isometric conditions for exerting the knee extensor forces.

\section{Instruction protocol}

During each experimental session, the participants performed one set of three MVCs with the "hard-and-fast" instruction and one set of three MVCs with the "fast" instruction in randomized order (Sahaly et al., 2001). The MVCs were four seconds in duration with a two-minute rest between contractions. A pilot test confirmed that the recovery duration between MVCs was sufficient to avoid neuromuscular fatigue. The participants were instructed to give maximal effort and to avoid countermovement. An additional MVC was performed in the event of a countermovement. The participants were given vigorous verbal encouragement during all MVCs with no feedback (Belkhiria et al., 2017). Each participant was informed of the number of MVCs and asked to avoid pacing (Halperin et al., 2014).

\section{Signal processing}

The force and EMG signals were sampled simultaneously at $1 \mathrm{kHz}$ using Biopac MP150 (Biopac System Inc., Santa Barbara, CA), and stored on a personal computer after A/D conversion. The signals were processed off-line using AcqKnowledge 4.3 Software (Biopac Systems Inc., Aero Camino, Goleta, CA, USA). The force signal was smoothed using a digital zero-phase-lag finite impulse response low-pass filter with cut-off frequency of $20 \mathrm{~Hz}$. The fullwave EMG signal was filtered using a digital filter with bandwidths of $10-500 \mathrm{~Hz}$. All subsequent analyses were completed on the filtered signals.

Force and EMG onsets during each MVC were identified using an automated method and checked visually by the same experienced investigator. For each muscle, the onset of EMG activity was determined as the first point of the signal exceeding the $95 \%$ confidence interval for baseline activity with minimum duration of $20 \mathrm{~ms}$ (Di Fabio, 1987; Inglis et al., 2013). The signal was rectified and numerically integrated for $20 \mathrm{~ms}$. Onset of force signal was defined as the point in the signal where the rate of change rose above $1 \%$ of the RFDmax (Gabriel et al., 2001; Inglis et al., 2013) (Figure 1).

$F_{\max }$ was assessed as the peak of force sustained during each MVC. Additionally, the

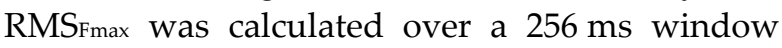
centered with the time point of $F_{\max }$. In order to examine the effect of instruction on the phases of explosive force production, the mean force output, elapsed time and RMS amplitude were calculated across five time intervals: between force onset and achieving 25\% of $\mathrm{F}_{\max }\left(\mathrm{T}_{0-25 \%}\right)$, between 25 and $50 \%$ of $F_{\max }\left(\mathrm{T}_{25-50 \%}\right)$, between 50 and $75 \%$ of $\mathrm{F}_{\max }\left(\mathrm{T}_{50-75 \%}\right)$, between 75 and $90 \%$ of $\mathrm{F}_{\max }\left(\mathrm{T}_{75-90 \%)}\right.$, and between $90 \%$ and $\mathrm{F}_{\max }\left(\mathrm{T}_{90-100 \%}\right)$ (Figure 1).

The RFDmax was computed from the differentiated force signal with a moving 20-ms window (Haff et al., 2015; Molina and Denadai, 2012; Penailillo et al., 2013; Sahaly et al., 2001) (Figure1) and was expressed in $\mathrm{N} \cdot \mathrm{s}^{-1}$. Additionally, the RMSRFDmax amplitude was calculated over a $128 \mathrm{~ms}$ window centered with the time point of the RFD max. 
The maximum value of the differentiated rectified EMG signal immediately after the EMG onset was calculated for each muscle to assess the rate of muscle activation (Inglis et al., 2013; Mitchell et al., 2011) (Figure 1). The highest value was defined as the maximal rate of muscle activation (RMAmax). The electromechanical delay defined as the time difference between the EMG onset and force onset was determined for each muscle (Figure 1). The maximal electromechanical delay $\left(E M D_{\max }\right)$, i.e., the largest electromechanical delay of the three muscles, was determined for each MVC (Zhou et al., 1995, 1996).

\section{Statistical analysis}

Assumptions for normality of distribution for all variables were checked and confirmed using the Shapiro-Wilk test. Dependent variables for each participant were averaged across the main three MVCs before group values were generated for each session. The effect of instruction and session on the dependent variables was analyzed with a two-way repeated measure ANOVA [Instruction (2) $\times$ Session (2)]. Likewise, the effect of instruction on the phases of explosive force production was studied using a two-way repeated measure ANOVA [Instruction (2) $\times$ Phase (5)]. The location of possible differences was assessed by a Bonferroni post hoc test. The standard error of measurement expressed in relative terms through the coefficient of variation $(\mathrm{CV})$, the intraclass correlation coefficient (ICC), and the corresponding 95\% confidence interval $(95 \% \mathrm{CI})$ was calculated to assess the reliability of the measured variables.

The magnitudes of differences between instructions were expressed as standardized mean differences (Cohen's d effect size) with corresponding 95\% CIs (Cohen, 1992). The criteria to interpret the magnitude of the effect sizes were 0-0.19 (trivial), 0.2-0.49 (small), $\quad 0.50-0.79$ (medium), and $>0.79$ (large).

The effect of instruction on the relationship between the RFD $\mathrm{max}_{\text {ax }} \mathrm{RMA}_{\max }, \mathrm{EMD}_{\max }$ and RMSRFDmax was studied by multiple linear regression according to the following model:

$R F D_{\max }=\mathrm{a}+\mathrm{b} \cdot \mathrm{x}+\mathrm{c} \cdot \mathrm{I}+\varepsilon$

where " $x$ " was the independent variable, i.e., RMAmax or EMD max $_{\text {max }}$ or RMSRFDmax, "I" was a dummy variable corresponding to instruction ( $\mathrm{I}=$ 0 for the "fast" instruction and I = 1 for the "hardand-fast" instruction) and $\varepsilon$ was the error.
Data are presented as mean and standard deviation (mean $\pm \mathrm{SD}$ ) in the text and tables, but the figures show mean and standard error of the mean (mean $\pm \mathrm{SE}$ ). Statistical procedures were carried out using Statistica Software 10 (StatSoft, Maisons-Alfort, France), and the significance level was set at $p<0.05$.

\section{Results}

\section{Explosive force and neuromuscular parameters}

The descriptive statistics of the recorded data and the corresponding CV and ICC for both instructions are presented in Table 1. The analysis revealed no significant interaction effect between the instruction and session for all measured variables (all $p>0.05$ ). There was no significant main effect of session for all measured variables, except for the RMSMRFDmax $\left(\mathrm{F}_{1,19}=5.49, p=0.030\right)$, and no significant main effect of instruction was observed on $\mathrm{F}_{\max }\left(\mathrm{F}_{1,19}=0.04, p=0.84\right)$. Moreover, the RMSFmax tended to be higher with the "hardand-fast" instruction compared to the "fast" instruction $\left(\mathrm{F}_{1,19}=4.10, p=0.057\right)$.

A significant main effect of instruction was observed in the RFDmax $\left(F_{1,19}=50.47, p<\right.$ $0.001)$. The post hoc test revealed that the RFDmax was significantly higher with the "fast" instruction compared to the "hard-and-fast" instruction $(+36.07 \pm 10.63 \%, p<0.001)$. Likewise, a significant main effect of instruction was observed in the RMSRFDmax $\left(\mathrm{F}_{1,19}=5.97, p=0.024\right)$. The post hoc test revealed that the RMSRFDmax was significantly higher with the "fast" instruction than with the "hard-and-fast" instruction $(+15.10$ $\pm 12.93 \%, p=0.024$ ).

There was a significant main effect of instruction on the RMAmax $\left(\mathrm{F}_{1,19}=27.88, p<0.001\right)$ and $\mathrm{EMD}_{\max }\left(\mathrm{F}_{1,19}=5.44, p=0.031\right)$. The post hoc test revealed that the RMAmax was significantly higher with the "fast" instruction compared to the "hard-and-fast" instruction (+37.24 $\pm 14.76 \%, p<$ 0.001 ), whereas $E^{2} D_{\max }$ was significantly lower with the "fast" instruction than with the "hardand-fast" instruction $(-3.79 \pm 2.49 \%, p=0.030)$.

A significant main effect of instruction was observed in $\mathrm{T}_{25-50 \%}\left(\mathrm{~F}_{1,19}=36.04, p<0.001\right)$. The post hoc test revealed that the time elapsed at the $\mathrm{T}_{25-50 \%}$ phase was significantly lower with the "fast" instruction compared to the "hard-andfast" instruction $(-50.21 \pm 17.50 \%, p<0.001)$.

The Cohen's $d$ effect sizes for the 
difference between the "fast" and "hard-and-fast" instructions are displayed in Figure 2.

\section{Phases of voluntary force}

The average time, force and RMS measured across the phases of force development are displayed in Figure 3. The analysis of the time elapsed in each phase of force development showed a significant main effect of interval $\left(\mathrm{F}_{4,76}=\right.$ 103.68, $p<0.001)$. The post hoc test showed that the time elapsed at the phase $\mathrm{T}_{90-100 \%}$ was significantly higher than at the preceding phases $(p<0.001)$. Likewise, the time elapsed at the phase T55-90\% was significantly higher than at the preceding phases $(p<0.05)$. No significant effect of instruction $\left(\mathrm{F}_{1,19}=0.03, p=0.87\right)$ or interaction between the instruction and interval $\left(\mathrm{F}_{4,76}=1.57, p\right.$ $=0.19$ ) was observed in this variable.

The analysis of mean force production across the phases showed a significant main effect of interval $\left(\mathrm{F}_{4,76}=637.48, p<0.001\right)$. The post hoc test showed that mean force increased significantly across the phases $(p<0.001)$. No significant effect of instruction $\left(\mathrm{F}_{1,19}=0.01, p=\right.$ 0.92 ) or interaction effect between instruction and interval $\left(\mathrm{F}_{4,76}=0.15, p=0.96\right)$ was observed in this variable.

The analysis of RMS amplitude across the phases of force development showed a significant main effect of instruction $\left(\mathrm{F}_{1,19}=4.89, p=0.03\right)$ and interval $\left(\mathrm{F}_{4,76}=15.77, p<0.001\right)$, and an interaction effect between the instruction and interval $\left(\mathrm{F}_{4,76}=\right.$ $12.22, p<0.001)$. The post hoc test showed that the RMS amplitude was significantly higher at the phases $\mathrm{T}_{0-25 \%}$ (ES $=0.85$ [0.45-1.25], Rating: Large) and $\mathrm{T}_{25-50 \%}(\mathrm{ES}=0.57$ [0.23-0.90], Rating: Medium) with the "fast" instruction compared to the "hardand-fast" instruction $(p<0.001)$.

Relationship between force development and neuromuscular variables

The relationships of the RFD max with the RMAmax $_{\text {m }}$ EMD $_{\max }$ and RMS are displayed in Figure 4. A significant positive correlation was observed between the RFD max and RMAmax with a significant effect of instruction:

$\mathbf{R F D}_{\max }=4499.04+\left(0.11 \cdot \mathrm{RMA}_{\max }\right)-(1364.69 \cdot \mathrm{I})$

$\mathbf{R}=0.80, p=0.002$ for RMAmax and $p<0.001$ for I

A significant negative correlation was observed between the RFD max and EMDmax with a significant effect of instruction:

$\mathrm{RFD}_{\max }=8368.68-\left(62.16 \cdot \mathrm{EMD}_{\max }\right)-(1652.22 \cdot \mathrm{I})$

$\mathrm{R}=0.78, p=0.012$ for $\mathrm{EMD}_{\max }$ and $p<0.001$ for I
A significant positive correlation was observed between the RFDmax and RMS with a significant effect of instruction:

$\mathrm{RFD}_{\max }=4931.81+(2.95 \cdot \mathrm{RMS})-(1640.53 \cdot \mathrm{I})$

$\mathrm{R}=0.77, p=0.023$ for RMS and $p<0.001$ for I

\section{Discussion}

The aim of the present investigation was to examine the effect of a verbal instruction on explosive force production and between-session measurement reliability during maximal voluntary contractions of knee extensors. The main finding of this study was that the instruction impacted the RFDmax and associated neuromuscular variables (i.e., EMD $\mathrm{max}_{\text {ma }} \mathrm{RMA}_{\max }$ and RMS). Moreover, the reliability of the measurements was higher with the "fast" instruction compared to the "hard-and-fast" instruction.

Both $\mathrm{F}_{\max }$ and RFD $\max$ measurements are important for explosive movement assessment. In accordance with previous studies (Bemben et al., 1990; Bozic et al., 2012; Holtermann et al., 2007; Sahaly et al., 2001), these measurements were sensitive to the instructions given to the participant by the investigator prior to the contraction task. Bemben et al. (1990) compared $\mathrm{F}_{\max }$ and the RFDmax during isometric handgrip contraction tasks. The authors showed that $\mathrm{F}_{\max }$ was significantly higher when participants were instructed to reach $\mathrm{F}_{\max }$ as quickly as possible by squeezing hard and fast (i.e., "hard-and-fat" instruction), whereas the RFD $\max$ was significantly higher when participants were instructed to squeeze as fast as possible without any concern into $F_{\max }$ (i.e., "fast" instruction). In another study, Sahaly et al. (2001) showed that the RFD $\max$ was significantly higher $(20-46 \%)$ when participants were instructed to push as "fast" as possible compared when participants were instructed to push "hard-and-fast" during isometric elbow flexion and leg press exercises. However, the authors reported no significant effect of instruction on $\mathrm{F}_{\max }$ during these tasks. Likewise, Holtermann et al. (2007) reported a significant difference of the RFDmax between the "fast" and the "hard-and-fast" instruction during isometric contractions of the plantar flexors $(30.5 \%, p<$ $0.01)$. However, no significant difference was observed for $F_{\max }$ between the two instructions $(0.8 \%, p>0.05)$. In the current study, $\mathrm{F}_{\max }$ was 
similar between both instructions. Nevertheless, there was a trend of the RMS at $\mathrm{F}_{\max }$ to be lower with the "fast" instruction compared to the "hard- and-fast" instruction suggesting possible difference in neuronal strategy within the same contraction.

Table 1

Descriptive statistics of measured variables

\begin{tabular}{|c|c|c|c|c|c|c|}
\hline & & \multicolumn{2}{|c|}{ "hard-and-fast" instruction } & \multicolumn{2}{|c|}{ "fast" instruction } & \multirow{2}{*}{ Global effect } \\
\hline & & Test & Retest & Test & Retest & \\
\hline \multirow[t]{3}{*}{$F_{\max }(\mathrm{N})$} & Mean \pm SD & $697.7 \pm 117.2$ & $693.4 \pm 120.5$ & $698.4 \pm 128.6$ & $688.6 \pm 128.7$ & \multirow{3}{*}{$\begin{array}{l}\text { Instruction: NS } \\
\text { Session: NS } \\
\text { Interaction: NS }\end{array}$} \\
\hline & CV $(95 \%$ CI $)$ & \multicolumn{2}{|c|}{$3.6(2.7-5.2)$} & \multicolumn{2}{|c|}{$2.8(2.1-4.1)$} & \\
\hline & ICC $(95 \%$ CI $)$ & \multicolumn{2}{|c|}{$0.96(0.91-0.99)$} & \multicolumn{2}{|c|}{$0.98(0.95-0.99)$} & \\
\hline \multirow[t]{3}{*}{$\mathrm{RMS}_{\mathrm{Fmax}}(\mu \mathrm{V})$} & Mean \pm SD & $352.5 \pm 114.8$ & $350.8 \pm 123.5$ & $333.2 \pm 192.0$ & $319.6 \pm 98.8$ & \multirow{3}{*}{$\begin{array}{l}\text { Instruction: } 0.057 \\
\text { Session: NS } \\
\text { Interaction: NS }\end{array}$} \\
\hline & CV $(95 \% \mathrm{CI})$ & \multicolumn{2}{|c|}{$6.4(4.8-9.4)$} & \multicolumn{2}{|c|}{$9.1(6.8-13.5)$} & \\
\hline & ICC $(95 \%$ CI $)$ & \multicolumn{2}{|c|}{$0.97(0.94-0.99)$} & \multicolumn{2}{|c|}{$0.93(0.83-0.97)$} & \\
\hline \multirow[t]{3}{*}{$R F D_{\max }\left(\mathrm{N} \cdot \mathrm{s}^{-1}\right)$} & Mean \pm SD & $3917 \pm 907$ & $4053 \pm 855$ & $5880 \pm 1066$ & $5597 \pm 808$ & \multirow{3}{*}{$\begin{array}{l}\text { Instruction: }{ }^{*} \\
\text { Session: NS } \\
\text { Interaction: NS }\end{array}$} \\
\hline & CV $(95 \%$ CI $)$ & \multicolumn{2}{|c|}{$16.2(12.1-24.5)$} & \multicolumn{2}{|c|}{$7.3(5.5-10.9) \S$} & \\
\hline & ICC (95\% CI) & \multicolumn{2}{|c|}{$0.56(0.16-0.80)$} & \multicolumn{2}{|c|}{$0.84(0.64-0.93) \S$} & \\
\hline \multirow[t]{3}{*}{$\operatorname{RMSRFDmax}_{(\mu \mathrm{V})}$} & Mean \pm SD & $239.4 \pm 95.2$ & $230.4 \pm 102.9$ & $281.9 \pm 105.2$ & $264.5 \pm 114.3$ & \multirow{3}{*}{$\begin{array}{l}\text { Instruction: }{ }^{*} \\
\text { Session: }{ }^{*} \\
\text { Interaction: NS }\end{array}$} \\
\hline & CV $(95 \% \mathrm{CI})$ & $9.6(7.2$ & $4.3)$ & $10.4(7$ & 15.5) & \\
\hline & ICC (95\% CI) & $0.95(0.8$ & $0.98)$ & $0.93(0$. & $0.97)$ & \\
\hline \multirow[t]{3}{*}{$\begin{array}{l}\mathrm{RMA} \max \left(\mu \mathrm{V} \cdot \mathrm{s}^{-}\right. \\
\left.{ }^{1}\right)\end{array}$} & Mean \pm SD & $7986 \pm 3690$ & $7963 \pm 4185$ & $11854 \pm 3874$ & $11394 \pm 3855$ & \multirow{3}{*}{$\begin{array}{l}\text { Instruction: }{ }^{* *} \\
\text { Session: NS } \\
\text { Interaction: NS }\end{array}$} \\
\hline & CV $(95 \%$ CI $)$ & \multicolumn{2}{|c|}{$22.3(16.6-34.2)$} & \multicolumn{2}{|c|}{$9.8(7.3-14.6) \S$} & \\
\hline & ICC $(95 \%$ CI $)$ & \multicolumn{2}{|c|}{$0.85(0.65-0.94)$} & \multicolumn{2}{|c|}{$0.94(0.85-0.98) \S$} & \\
\hline \multirow[t]{3}{*}{$\mathrm{EMD}_{\max }(\mathrm{ms})$} & Mean \pm SD & $44.53 \pm 5.97$ & $43.37 \pm 5.03$ & $42.28 \pm 6.56$ & $42.35 \pm 6.12$ & \multirow{3}{*}{$\begin{array}{l}\text { Instruction: * } \\
\text { Session: NS } \\
\text { Interaction: NS }\end{array}$} \\
\hline & CV $(95 \%$ CI) & $7.0(5.3$ & $0.4)$ & $10.3(7$ & 15.4) & \\
\hline & ICC $(95 \%$ CI $)$ & $0.74(0$. & 0.89) & $0.61(0$. & 0.83) & \\
\hline \multirow[t]{3}{*}{$\mathrm{T}_{25-50 \%}(\mathrm{~ms})$} & Mean \pm SD & $60.62 \pm 23.29$ & $57.53 \pm 16.01$ & $33.97 \pm 9.03$ & $36.77 \pm 10.94$ & \multirow{3}{*}{$\begin{array}{l}\text { Instruction: }{ }^{* *} \\
\text { Session: NS } \\
\text { Interaction: NS }\end{array}$} \\
\hline & CV $(95 \%$ CI $)$ & $24.6(18$ & 37.9) & $9.5(7.1$ & $4.1) \S$ & \\
\hline & ICC $(95 \%$ CI $)$ & $0.65(0.3$ & $0.84)$ & $0.90(0.7$ & $0.96) \S$ & \\
\hline
\end{tabular}

$F_{\max }$ : maximal voluntary force; $R M S_{F \max }$ RMS amplitude at $F_{\max }$ RFD $D_{\max }$ : maximal rate of force development; $R M S_{R F D \max }$ RMS amplitude at RFD max; RMA max: maximal rate of muscle activity;

EMD max: maximal electromechanical delay; $T_{25-50 \%}$ : time elapsed between achieving $25 \%$ and $50 \%$ of $F_{\max }$; CV: coefficient of variation; ICC: intraclass correlation coefficient;

95\% CI: 95\% confidence interval.

§: Above (ICC) or below (CV) the $95 \%$ CI observed from the hard-and-fast instruction

${ }^{*}: p<0.05,{ }^{* *}: p<0.001$, NS: non-significant $(p>0.05)$ 

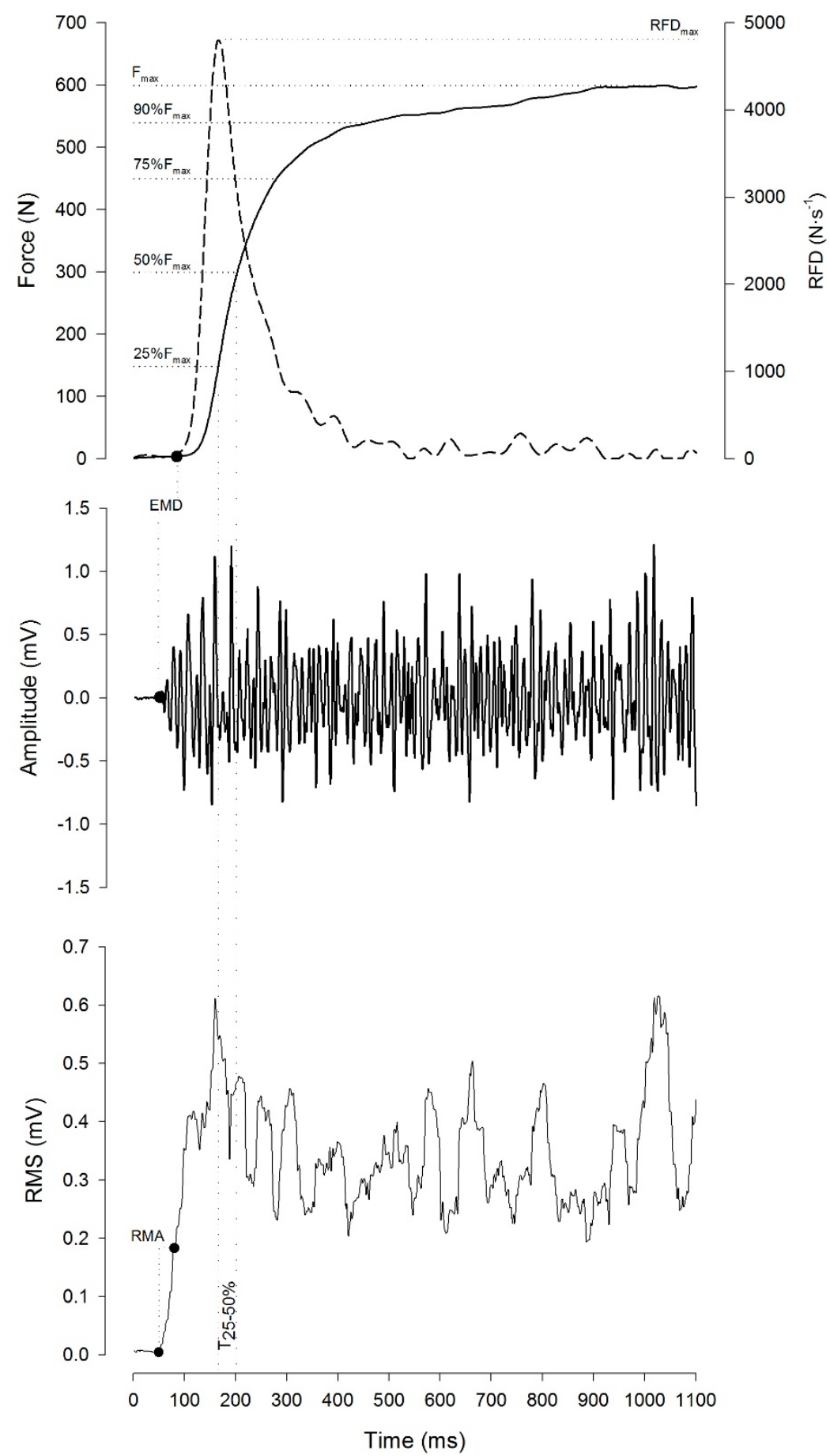

Figure 1

An example of force-time and EMG-time curves recorded during a maximal voluntary contraction (MVC) with the "fast" instruction. Fmax: maximal voluntary force; RFD max: maximal rate of force development; EMD: electromechanical delay; RMA: rate of muscle activity. The mean force output, elapsed time and RMS amplitude were calculated across five time intervals: between the force onset and achieving $25 \%$ of $F_{\max }\left(T_{0-25 \%}\right)$, between 25 and $50 \%$ of $F_{\max }\left(T_{25-50 \%}\right)$, between 50 and $75 \%$ of $F_{\max }\left(T_{50-75 \%}\right)$, between 75 and $90 \%$ of $F_{\max }\left(T_{75-90 \%}\right)$, and between $90 \%$ of $F_{\max }$ and $F_{\max }\left(T_{90-100 \%}\right)$. 


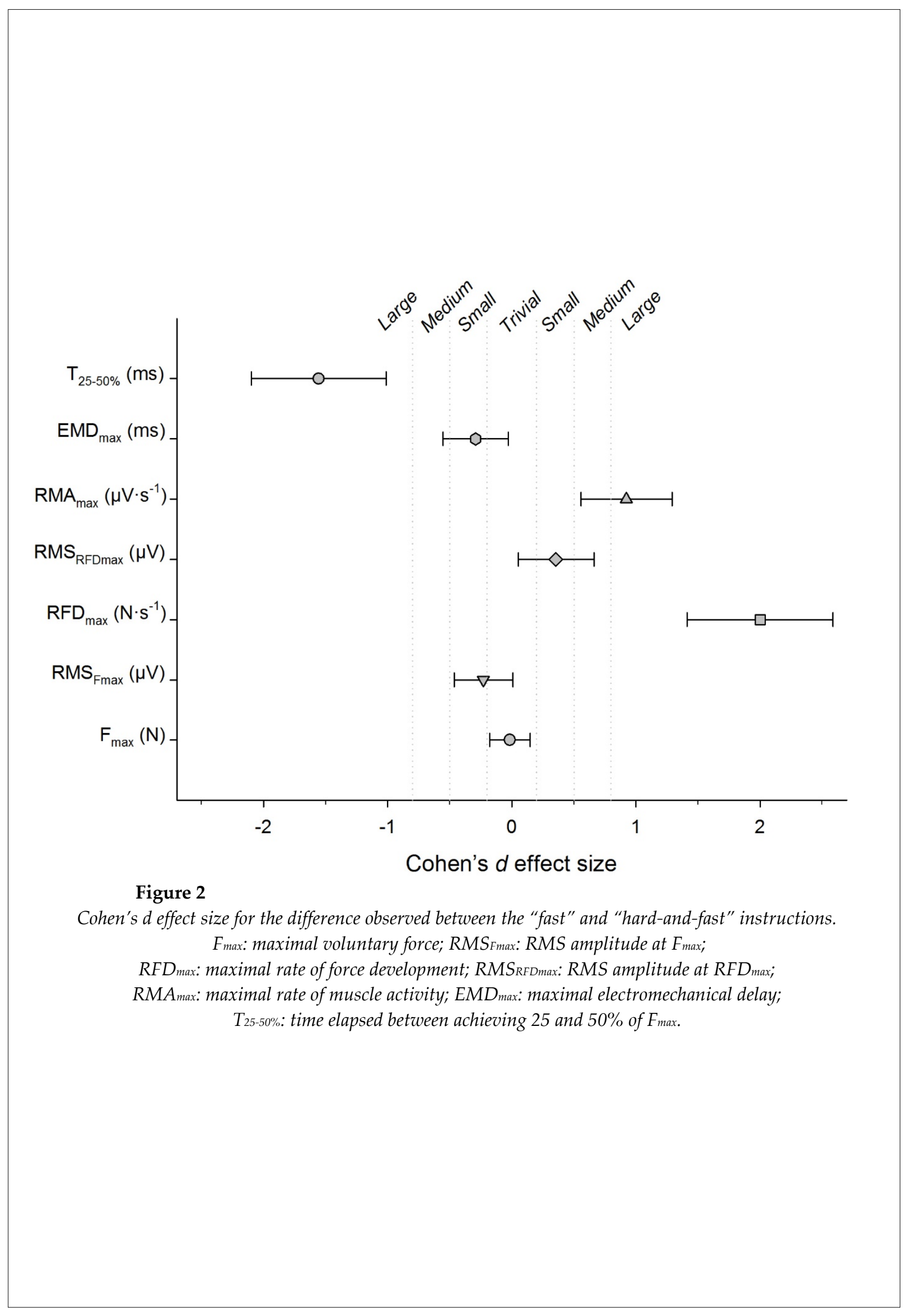



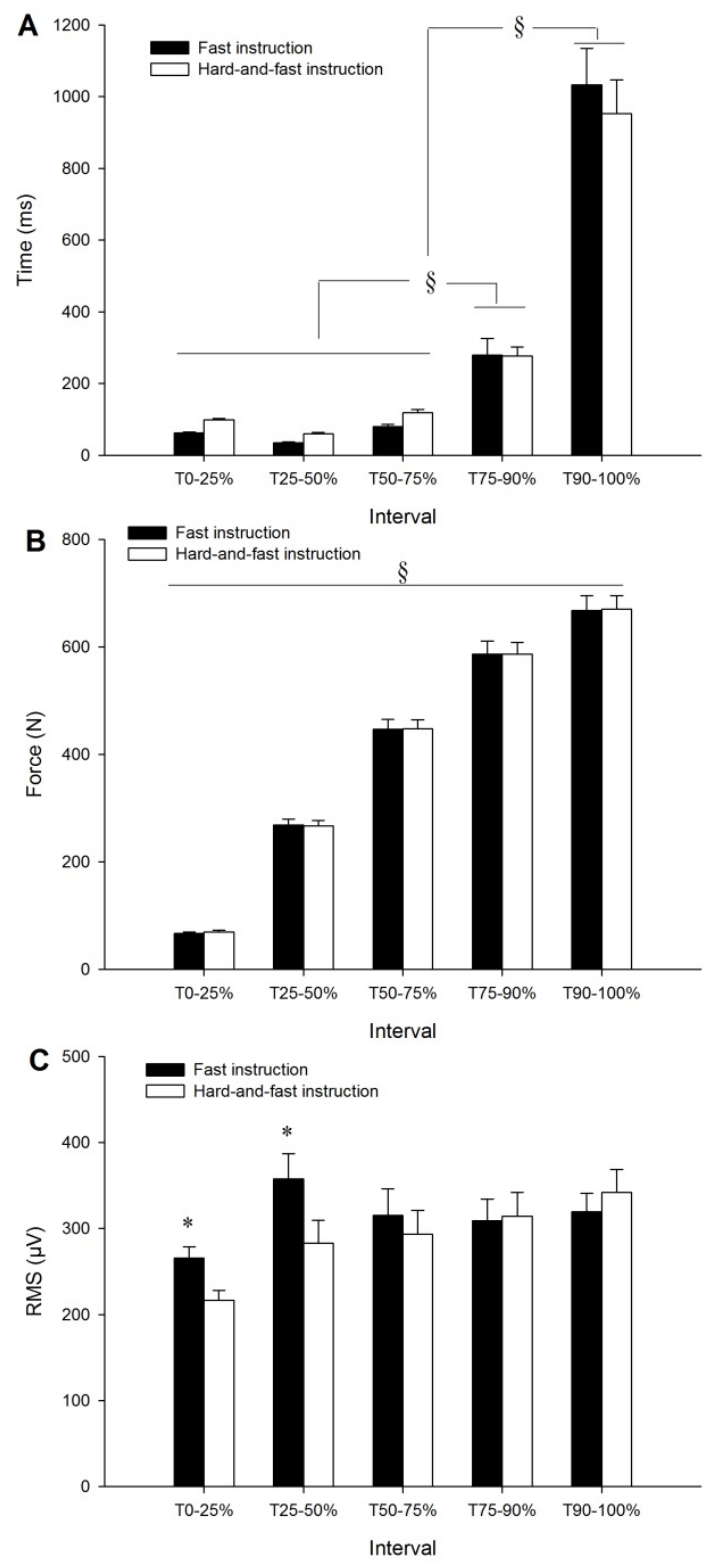

Figure 3

Mean time (A), force production (B) and RMS amplitude (C) across the phases of force development from the contraction onset $\left(T_{0}\right)$ to $F_{\max }\left(T_{100 \%}\right)$.

Data show mean $\pm S E$ of the two sessions. §: increased significantly among the different phases $(p<0.05)$. *: Significantly higher with the "fast" instruction compared to the "hard-and-fast" instruction $(p<0.001)$. 

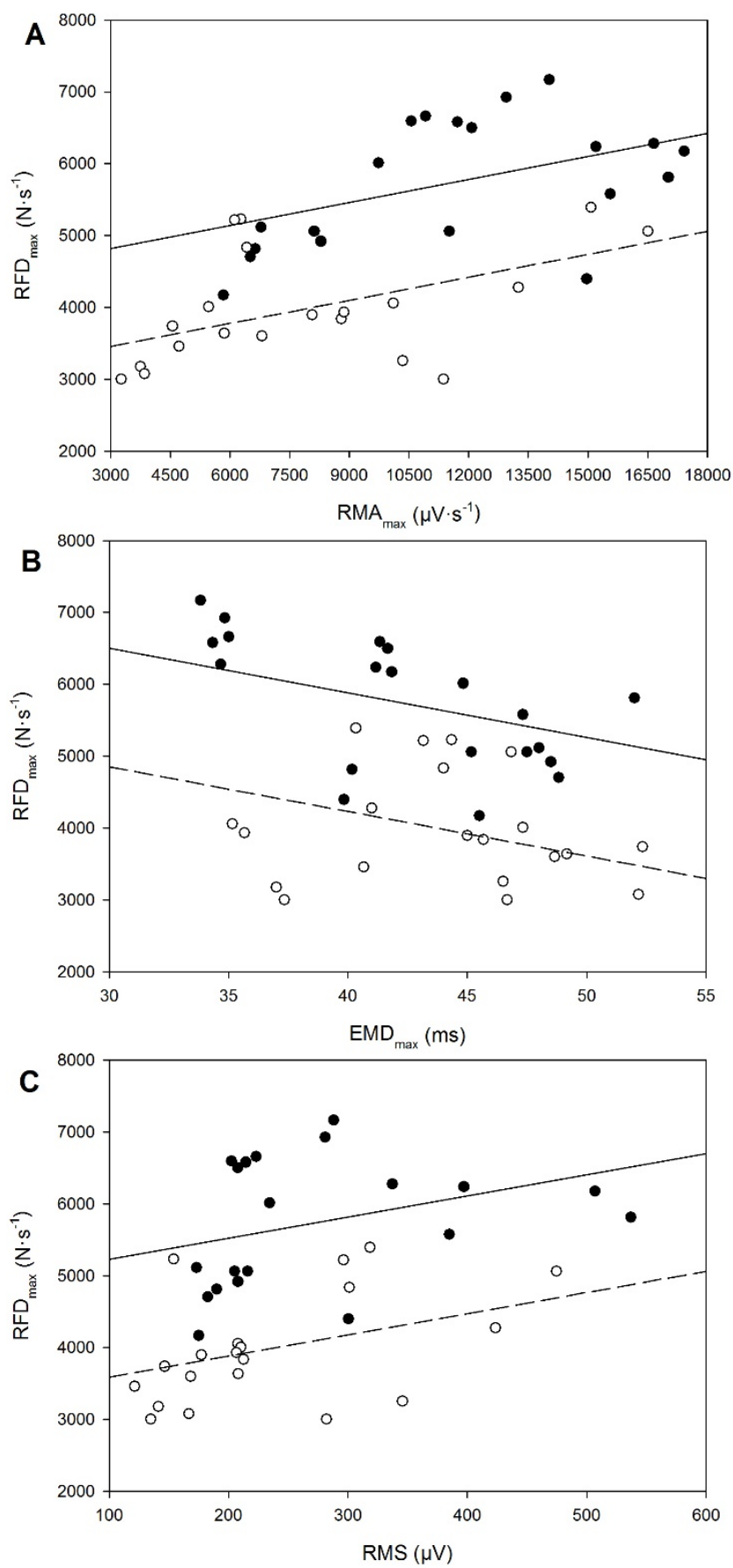

\section{Figure 4}

Maximal rate of force development $\left(R F D_{\max }\right)$ plotted against the following variables:

$A$, maximal rate of muscle activation ( $\left.R M A_{\max }\right) ; B$, maximal electromechanical delay (EMD $D_{\max }$ ); and $C$, root mean square (RMS). A solid line and black symbols correspond to the "fast" instruction, whereas a dashed line

and empty symbols correspond to the hard-and-fat instruction. Data are the mean of both sessions. 
In accordance with the previous studies (Holtermann et al., 2007; Sahaly et al., 2001), the RFDmax in the current study was higher with the "fast" instruction compared to the "hard-andfast" instruction (36.07 $\pm 10.63 \%)$ with large effect size $(2.0 \pm 0.59)$. Interestingly, the higher $\mathrm{RFD}_{\max }$ values observed with the "fast" instruction were associated with greater muscle activation of the agonist muscles during the phase of force development (i.e., from the contraction onset to achieving $50 \%$ of $\mathrm{F}_{\max }$ ). These findings were most likely due to a central factor since the RFDmax was primarily a factor of agonist neural drive. During the activation of knee extensors, their motor neurons are facilitated by motor commands from supraspinal centers via the corticospinal tract. Because the participants were asked to voluntarily extend the knee as fast as possible against the force transducer, this would invoke a motor command from the supraspinal centers (Johnson et al., 2015). The higher values of the RFDmax with the "fast" instruction were concomitant with higher values of the RMAmax and RMS amplitude. The relations within the RFDmax-RMAmax and RFD max-RMS were significantly positive.

The rate of rise of the muscle's activity (i.e., RMA) at its onset may be affected by earlier motor unit recruitment, a higher discharge rate and a higher rate of doublet discharge, defined as two proximate spikes, less than $5 \mathrm{~ms}$ apart (Van Cutsem et al., 1998). Therefore, a higher RMAmax involves higher motor unit discharge rates. Indeed, the RMA is controlled by the muscle fiber action's potential conduction velocity, which has been shown to relate linearly to the EMG power spectrum (Lindstrom et al., 1970; Stulen and DeLuca, 1981). Moreover, the activation of the fibers influences the rate of cross bridge cycling. If the individual sarcomeres within a muscle fiber can be activated more quickly, the shortening velocity of the whole fiber would be enhanced (Wickiewicz et al., 1984). Indeed, in the current study, the time elapsed in the phase $\mathrm{T}_{25-50 \% \text {, in }}$ which the RFDmax occurs, was significantly lower with the "fast" instruction compared to the "hardand-fast" one.

The relation within the RFD max EMD $_{\max }$ was significantly negative. During skeletal muscle contractions, EMD reflects both electrochemical and mechanical processes (Cavanagh and Komi, 1979; Zhou et al., 1995). Electrochemical processes include synaptic transmission, action potential propagation across the sarcolemma, excitationcontraction coupling process, $\mathrm{Ca}^{2+}$ release into the sarcoplasm, with subsequent $\mathrm{Ca}^{2+}$ binding to active sites and the formation of cross-bridges. Mechanical processes include force transmission along the active and passive parts of the series elastic component and stretching of the series elastic component by the contractile elements (Cavanagh and Komi, 1979; Zhou et al., 1995). Therefore, any factor influencing these processes may influence EMD. However, the time course of the stretching of the series elastic component by the contractile component is considered a major portion of EMD time (Cavanagh and Komi, 1979). The changes in electrochemical rather than mechanical processes could be explained by the lower EMD values with the "fast" instruction.

Reliability refers to the consistency of a measurement which can be assessed by various methods (Weir, 2005). Generally the first step when testing the reliability is to examine statistically the difference between the two measures (Weir, 2005). In the present study, there were no statistical differences between test and retest sessions for any of the measured variables. However, statistical bias does not give a sensitive indication of random variation between measures. Therefore, Weir (2005) recommends quantifying reliability by means of the ICC and CV. The ICC is considered as high for values above 0.90, moderate for values between 0.80 and 0.90 , and low for values below 0.80 (Vincent, 2004). The reliability of $\mathrm{F}_{\max }$ in the present study was high for both instructions. These findings were in agreement with previous studies on the reliability of $\mathrm{F}_{\max }$ during various MVC tasks (Bemben et al., 1990; Blazevich et al., 2002; Maffiuletti et al., 2007; Mirkov et al., 2004).

Furthermore, the reliability of $F_{\max }$ was somewhat higher than of the RFDmax. These observations may be due to some methodological issues regarding the determination of the RFDmax. Indeed, various sampling windows have been proposed for the assessment of the RFDmax. The differentiated force signal with a moving 20-ms window was the most used method to assess the RFD $_{\max }$ (Haff et al., 2015; Molina and Denadai, 2012; Penailillo et al., 2013; Sahaly et al., 2001). In a reliability study, Haff et al. (2015) reported high reliability of a $20 \mathrm{~ms}$ sampling window (ICC = 
$0.90,90 \% \mathrm{CI}=[0.73-0.97] ; \mathrm{CV}=12.9 \%, 90 \% \mathrm{CI}=$ [0.5-20.7]) compared to 2, 5, 10, 30 and $50 \mathrm{~ms}$ sampling windows during isometric mid-thigh clean pull tasks. In the current study, high variability of the RFDmax was observed with the "hard-and-fast" instruction compared to the "fast" instruction, suggesting the latter one is better for the RFDmax assessment. Based upon the data collected in the current study it is clear that strength and conditioning professionals need to carefully consider the instruction used to assess the RFD max during the MVCs as the present study demonstrates that the instruction impacts the reliability of the measurement. This becomes an increasingly important consideration when the purpose of the assessment is to monitor performance and track changes in neuromuscular performance over time particularly in individuals involved in sports activities in which explosive force production is fundamental.

\section{Conclusion}

The instruction given by the investigator prior to a MVC task impacts the reliability of neuromuscular performance. The instruction should emphasize the importance of contracting skeletal muscles as fast as possible to maximize the RFDmax. Moreover, attempting to achieve high values of $F_{\max }$ and the RFD max within the same MVC may result in low reliability of the measures. These findings have several practical implications for practitioners and researchers who are involved with both explosive movements and potentially injurious situations.

\section{References}

Belkhiria C, Driss T, Habas C, Jaafar H, Guillevin R, de Marco G. Exploration and Identification of CorticoCerebellar-Brainstem Closed Loop During a Motivational-Motor Task: an fMRI Study. Cerebellum, 2017; 16: 326-339

Bell DG. The influence of air temperature on the EMG/force relationship of the quadriceps. Eur J Appl Physiol Occup Physiol, 1993; 67: 256-260

Bellumori M, Jaric S, Knight CA. The rate of force development scaling factor (RFD-SF): protocol, reliability, and muscle comparisons. Exp Brain Res, 2011; 212: 359-369

Bemben MG, Clasey JL, Massey BH. The effect of the rate of muscle contraction on the force-time curve parameters of male and female subjects. Res Q Exerc Sport, 1990; 61: 96-99

Blazevich AJ, Gill N, Newton RU. Reliability and validity of two isometric squat tests. J Strength Cond Res, 2002; 16: 298-304

Bozic PR, Pazin N, Berjan B, Jaric S. Evaluation of alternating consecutive maximum contractions as an alternative test of neuromuscular function. Eur J Appl Physiol, 2012; 112: 1445-1456

Cavanagh PR, Komi PV. Electromechanical delay in human skeletal muscle under concentric and eccentric contractions. Eur J Appl Physiol Occup Physiol, 1979; 42: 159-163

Christ CB, Boileau RA, Slaughter MH, Stillman RJ, Cameron J. The effect of test protocol instructions on the measurement of muscle function in adult women. J Orthop Sports Phys Ther, 1993; 18: 502-510

Cohen J. A power primer. Psychol Bull, 1992; 112: 155-159

Di Fabio RP. Reliability of computerized surface electromyography for determining the onset of muscle activity. Phys Ther, 1987; 67: 43-48

Gabriel DA, Basford JR, An K. Training-related changes in the maximal rate of torque development and EMG activity. J Electromyogr Kinesiol, 2001; 11: 123-129

Gołaś A, Maszczyk A, Zajac A, Mikolajec K, Stastny P . Optimizing Post Activation Potentiation for Explosive Activities in Competitive Sports. J Hum Kinet, 2016; 52 (1): 95-106

Haff GG, Ruben RP, Lider J, Twine C, Cormie P. A comparison of methods for determining the rate of force development during isometric midthigh clean pulls. J Strength Cond Res, 2015; 29: 386-395 
Halperin I, Aboodarda SJ, Basset FA, Byrne JM, Behm DG. Pacing strategies during repeated maximal voluntary contractions. Eur J Appl Physiol, 2014; 114: 1413-1420

Hermens HJ, Freriks B, Disselhorst-Klug C, Rau G. Development of recommendations for SEMG sensors and sensor placement procedures. J Electromyogr Kinesiol, 2000; 10: 361-374

Holtermann A, Roeleveld K, Vereijken B, Ettema G. The effect of rate of force development on maximal force production: acute and training-related aspects. Eur J Appl Physiol, 2007; 99: 605-613

Inglis JG, Vandenboom R, Gabriel DA. Sex-related differences in maximal rate of isometric torque development. J Electromyogr Kinesiol, 2013; 23: 1289-1294

Jaafar H, Lajili H. Separate and combined effects of time of day and verbal instruction on knee extensor neuromuscular adjustments. Appl Physiol Nutr Metab, 2018; 43: 54-62

Johnson ST, Kipp K, Norcross MF, Hoffman MA. Spinal and supraspinal motor control predictors of rate of torque development. Scand J Med Sci Sports, 2015; 25: 623-629

Juneja H, Verma SK, Khanna GL. Isometric strength and its relationship to dynamic performance: a systematic review. J Exerc Sci Physio, 2012; 6: 60-69

Lindstrom L, Magnusson R, Petersen I. Muscular fatigue and action potential conduction velocity changes studied with frequency analysis of EMG signals. Electromyography, 1970; 10: 341-356

Maffiuletti NA, Aagaard P, Blazevich AJ, Folland J, Tillin N, Duchateau J. Rate of force development: physiological and methodological considerations. Eur J Appl Physiol, 2016; 116: 1091-1116

Maffiuletti NA, Bizzini M, Desbrosses K, Babault N, Munzinger U. Reliability of knee extension and flexion measurements using the Con-Trex isokinetic dynamometer. Clin Physiol Funct Imaging, 2007; 27: 346353

Mirkov DM, Nedeljkovic A, Milanovic S, Jaric S. Muscle strength testing: evaluation of tests of explosive force production. Eur J Appl Physiol, 2004; 91: 147-154

Mitchell C, Cohen R, Dotan R, Gabriel D, Klentrou P, Falk B. Rate of muscle activation in power- and endurance-trained boys. Int J Sports Physiol Perform, 2011; 6: 94-105

Molina R, Denadai BS. Dissociated time course recovery between rate of force development and peak torque after eccentric exercise. Clin Physiol Funct Imaging, 2012; 32: 179-184

Penailillo L, Silvestre R, Nosaka K. Changes in surface EMG assessed by discrete wavelet transform during maximal isometric voluntary contractions following supramaximal cycling. Eur J Appl Physiol, 2013; 113: 895-904

Sahaly R, Vandewalle H, Driss T, Monod H. Maximal voluntary force and rate of force development in humans--importance of instruction. Eur J Appl Physiol, 2001; 85: 345-350

Sahaly R, Vandewalle H, Driss T, Monod H. Surface electromyograms of agonist and antagonist muscles during force development of maximal isometric exercises--effects of instruction. Eur J Appl Physiol, 2003; 89: 79-84

Stastny P, Tufano J, Lehnert M, Zaatar A, Golas A, Xaverova Z, Masczyk A, Hip Abductors and Thigh Muscles Strength Ratio and Their Relation to Electromyography Amplitude during Split squat and Walking lunge exercise, Acta Gymnica 2015; 45(2): 51-59

Stulen FB, DeLuca CJ. Frequency parameters of the myoelectric signal as a measure of muscle conduction velocity. IEEE Trans Biomed Eng, 1981; 28: 515-523

Van Cutsem M, Duchateau J, Hainaut K. Changes in single motor unit behaviour contribute to the increase in contraction speed after dynamic training in humans. J Physiol, 1998; 513 (Pt 1): 295-305

Vincent W. Statistics in Kinesiology: Human Kinetics 2004: 328

Weir JP. Quantifying test-retest reliability using the intraclass correlation coefficient and the SEM. J Strength Cond Res, 2005; 19: 231-240 
Wickiewicz TL, Roy RR, Powell PL, Perrine JJ, Edgerton VR. Muscle architecture and force-velocity relationships in humans. J Appl Physiol Respir Environ Exerc Physiol, 1984; 57: 435-443

Zhou S, Lawson DL, Morrison WE, Fairweather I. Electromechanical delay in isometric muscle contractions evoked by voluntary, reflex and electrical stimulation. Eur J Appl Physiol Occup Physiol, 1995; 70: 138145

Zhou S, McKenna MJ, Lawson DL, Morrison WE, Fairweather I. Effects of fatigue and sprint training on electromechanical delay of knee extensor muscles. Eur J Appl Physiol Occup Physiol, 1996; 72: 410-416

\section{Corresponding author:}

\section{Dr. Hamdi Jaafar}

Institut du savoir Montfort - A Knowledge Institute

713 Montreal Rd, Ottawa, ON K1K 0T2, Canada

Tel.: +1-613-746-4621, ext. 6071

Fax: +1-613-288-1337

E-mail: hamdijaafar@montfort.on.ca 